\title{
Forma e função dos músculos perineais da viscacha (Lagostomus maximus) ${ }^{1}$
}

\author{
Naira C.G. Pieri' ${ }^{2}$, Alicia M. Flamini ${ }^{3}$, Claudio G. Barbeito ${ }^{3}$, Juliana B.Casals ${ }^{2}$, Beatriz \\ K. Roque ${ }^{4}$, Phelipe O. Favaron ${ }^{2}$, Maria Angelica Miglino ${ }^{2}$ e Daniele S. Martins ${ }^{4^{*}}$
}

\begin{abstract}
Pieri N.C.G., Alicia M. Flamini A.M., Barbeito C.G., Casals J.B., Roque K.B., Favaron P.O., Miglino M.A. \& Martins D.S. 2012. [Shape and function of the perineal muscles of viscacha (Lagostomus maximus).] Forma e função dos músculos perineais da viscacha (Lagostomus maximus). Pesquisa Veterinária Brasileira 32(2):183-187. Departamento de Zootecnia, Faculdade de Zootecnia e Engenharia de Alimentos, Universidade de São Paulo, Av. Duque de Caxias Norte 225, Pirassununga, SP 13635-900, Brazil. E-mail: daniele@usp.br.

Among the rodent species studied we can highlight the wide variation in the morphology of the male reproductive system. Thus, considering the ecological importance of rodents, and the large number and geographical representation of this animal, as well as shortages regarding the reproductive anatomy, we developed this study with viscacha, a South American histricomorph rodent. As this species has some very peculiar reproductive features, we described the gross anatomy of the perineal muscles and the role of copulatory behavior. The perineal region of viscacha is composed of five muscles, three of which are arranged in the superficial genitourinary diaphragm, as Musculus ischiocavernosus, M. bulbocavernosus and $M$. bulbospongiosus, and the muscles that lie at the pelvic diaphragm, M. levator ani and M. retractor penis. Therefore, we emphasize that the study of the pelvic floor in wild animals is of great value, then contribute to a better understanding of the mechanisms related to erection and ejaculation or collaborate with studies on the reproduction of animals.
\end{abstract}

INDEX TERMS: Perineal muscles, viscacha, Lagostomus maximus.

RESUMO.- Entre as espécies de roedores já estudadas podemos destacar a grande variação na morfologia do aparelho reprodutor masculino. Assim, considerando a importância ecológica dos roedores, e a grande representatividade numérica quanto geográfica destes animais, bem como a escassez que aborda a anatomia reprodutiva, desenvolvemos este trabalho com um roedor histricomorfo da América do $\mathrm{Sul}$ a viscacha. Este animal apresenta algumas características reprodutivas bem peculiares, desta forma descreveremos a anatomia macroscópica da musculatura perineal, e o papel dos músculos no comportamento copulatório des-

\footnotetext{
${ }^{1}$ Recebido em 30 de setembro de 2011.

Aceito para publicação em 23 de janeiro de 2012.

${ }^{2}$ Departamento de Cirurgia, Faculdade de Medicina Veterinária e Zootecnia, Universidade de São Paulo (USP), Av. Prof. Dr. Orlando Marques de Paiva 87, Cidade Universitária, São Paulo, SP 05508-270, Brasil.

${ }^{3}$ Facultad de Ciencias Veterinarias,Universidade Nacional de La Plata, Calle 60 y 118 s/n, Casilla de Correo 296, La Plata, Provincia de Buenos Aires, Argentina.

${ }^{4}$ Faculdade de Zootecnia e Engenharia de Alimentos, USP, Av. Duque de Caxias Norte 225, Pirassununga, SP 13635 900, Brasil. *Autor para correspondência: daniele@usp.br
}

ta espécie. A região perineal da viscacha é composta por cinco músculos, sendo que três músculos encontram-se dispostos no diafragama urogenital superficial, Musculus ischiocavernosus, M. bulbocavernosus e M. bulbospongiosus, e pelos músculos que se encontram no diafragma pélvico, $M$. levator ani e $M$. retractor penis. Sendo assim, destacamos que o estudo do assoalho pélvico em animais silvestres é de grande valia, uma vez que contribuem com o melhor entendimento dos mecanismos relacionados â ereção e ejaculação; ou seja colaboram com os estudos sobre a reprodução dos animais.

TERMOS DE INDEXAÇÃO: Perineo, viscacha, Lagostomus maximus.

\section{INTRODUÇÃO}

Atualmente muitos estudos com animais silvestres vêem sendo desenvolvidos, no entanto, trabalhos com roedores na grande maioria das vezes estão associados a levantamentos sistemáticos, de ocorrência de espécies em uma determinada área e a biologia básica, porém quando abordam a reprodução, focam-se mais em características do período 
reprodutivo, desenvolvimento embrionário comparativo e poucos tratam dos aspectos relativos á biologia da reprodução e anatomia destes animais (Franciolli et al. 2011).

A viscacha (Lagostomus maximus) é um roedor da subordem Histricomorfo da América do Sul. Na ordem dos roedores, representa $43 \%$ das 4.629 espécies reconhecidas encontradas na classe Mamalia, sendo os quais oferecem uma extraordinária variedade de adaptações ecológicas suportando os mais diversos tipos de climas e altitudes, podendo com isso apresentar grande diversidade funcional. L. maximus estão sendo usados como modelo para muitos projetos de pesquisas, dentre eles o estudo dos órgãos endócrinos devido a sua variabilidade fisiológica em relação ao fotoperiodo (Piezzi et al. 1984, 1985, Dominguez et al. 1987, Mohamed et al. 1994) e o ciclo reprodutivo (Llanos \& Crespo 1952, Jackson 1989, Flamini et al. 2009).

As caracteristicas anatomicas dos músculos nos fornece informação sobre o hábito alimentar, força e comportamento reprodutivo dos mamíferos (Aversi-Ferreira et al. 2006). Através da contração destes é possivel promover os movimentos, que são efetuados por células especilizadas, denominadas fibras musculares cuja energia latente é ou pode ser controlada pelo sistema nervoso (Manzanares 2007). Portando, a musculatura perineal é de extrema importância no processo reprodutivo, pois os músculos são ritmicamente ativo durante a ereção peniana e ejaculação em ratos, cães e seres humanos (Hart \& Melese-D'hospital 1983, Karacan et al. 1983, Yajima 1989, Onuf 1900).

Desta forma, a região perineal é definida como a parte do tronco abaixo do diafragma da pelve, que consiste em um espaço losângular com os mesmos limites que a abertura inferior da pelve. 0 centro tendíneo do períneo ou corpo perineal é uma massa fibro-muscular localizada no plano mediano entre o canal e o diafragma urogenital, com o qual se funde vários músculos e se prendem ao centro tendíneo. Estes são os músculos transversos superficiais e profundos do períneo, Musculus bulbospongiosus, M. levator ani, $M$. sphincter ani externus e o músculo liso da túnica longitudinal do reto. Além destes músculos, as fáscias superficial e profunda do períneo e as fáscias superior e inferior do diafragma urogenital prendem-se a ele (Gray et al.1988) e são importantes na sustentação das vísceras pélvicas.

Existe ainda um segundo grupo de músculos perineais que são: o diafragma urogenital o qual ocupa o períneo anterior e se distinguem em músculos profundos e músculos superficiais, sendo eles: $M$. transversus perinei superficialis, M ischiocavernosus e M. bulbospongiosus (Watanabe 2000).

Em roedores a morfologia e função dos músculos perineais, vem sendo estudadas, por estes serem estriados, mais desenvolvidos e também por apresentarem músculos relacionados com a uretra, e aos mecanismos da reprodução, como o bulbospongiosus, ischiocavernosus e levator ani (Hayes 1965, McKenna \& Nadelhaft 1986, Tullber 1899, Holmes \& Sachs 1994, Jordan 1997, Poortmans \& Wyndaele 1998).

Assim, considerando a importância ecológica dos roedores, e a grande representatividade numérica quanto geográfica destes animais, bem como a escassez que aborda a anatomia reprodutiva, procuramos desenvolver o presente trabalho a fim de descrever a anatomia macroscópica da musculatura perineal, e destacar a importância do papel dos músculos no comportamento copulatório desta espécie.

\section{MATERIAL E MÉTODOS}

O estudo foi conduzido apos a aprovação da comissão de Ética em experimentação animal da Faculdade de Zootecnia e Engenharia de Alimento (FZEA/USP) e da Universidade Nacional de La Plata.

Para esta pesquisa, utilizamos dois machos adultos de Viscachas procedentes da Facultad de Ciencias Veterinarias, Univesidad Nacional de La Plata. Os exemplares foram capturados nas províncias de Buenos Aires, e mantidos em cativeiro com água e comida ad libidum. Em seguida foram anestesiados com cetamina e xilazina e posteriormente pesados em balança KERN 430-53 max $6000 \mathrm{~g} \mathrm{~d}=1 \mathrm{~g}$, após realizados os exames de anamese, mensuração e coleta de sangue; os animais foram eutanásiados seguindo o protocolo de Flamini et al. (2011) para viscachas.

Para coleta dos dados deste estudo fez-se uma abertura na região da rafe mediana do perineo para visualização das estruturas e musculatura da região alvo, por meio de observação das inserções proximais e distais. Os resultados obtidos foram documentados com câmera fotográfica digital (Sony Cyber-shot, 7.1 megapixels) e comparados com dados já existentes na literatura. Todas as designações das estruturas anatômicas dos músculos componentes da região perineal da Viscacha, basearam-se nas indicações do International Committee on Veterinary Anatomy.

\section{RESULTADO E DISCUSSÃO}

O períneo da viscacha consiste na parte do tronco caudal ao diafragma da pelve, formando uma região losângular (Fig.1A). As delimitações ósseas perineais são formadas pelos ossos interpúbico, ramos do ísquio, cóccix e tuberosidade isquiática, bem como descrito por Moore \& Dalley (1994) em períneo humano. Através da dissecação da linha média, evidenciamos a região perineal da viscaha composta por quarto músculos, sendo que três encontra-se dispostos no diafragama urogenital superficial, composto pelo Musculus ischiocavernosus, bulbocavernosus e bulbospongiosus; bem como os músculos do diafragma pelvico, os quais destacamos o levator ani e o retractor penis (Fig.2A,B).

Segundo Gray et al. (1988) e Moore \& Dalley (1994) o triângulo urogenital se dispõe em camadas, denominadas de fáscias, as quais se subdividem em: superficial do períneo, fáscia profunda do períneo (Fig.1B,C), fáscia inferior e superior do diafragma urogenital; tais estruturas estão fixadas lateralmente aos ramos do ísquio-púbico, enquanto que posteriormente fundem-se umas com as outras na borda posterior do diafragma urogenital. Em nosso estudo com a viscacha destacamos a fáscia profunda do períneo, onde notamos a presença dos músculos mais superficiais como bulbospongiosus e ischiocavernosus, ou seja, delimitando superficialmente o espaço perineal, no qual estão contidas as estruturas que formam a raiz do pênis e as fáscias inferior e superior do diafragma urogenital, as quais delimitam o espaço perineal profundo, no qual observamos, as glândulas bulbo-uretrais (Fig.2A).

Três músculos apresentam-se aderidos ao pênis da viscacha; um par de $M$. ischiocavernosus, que se estende sime- 

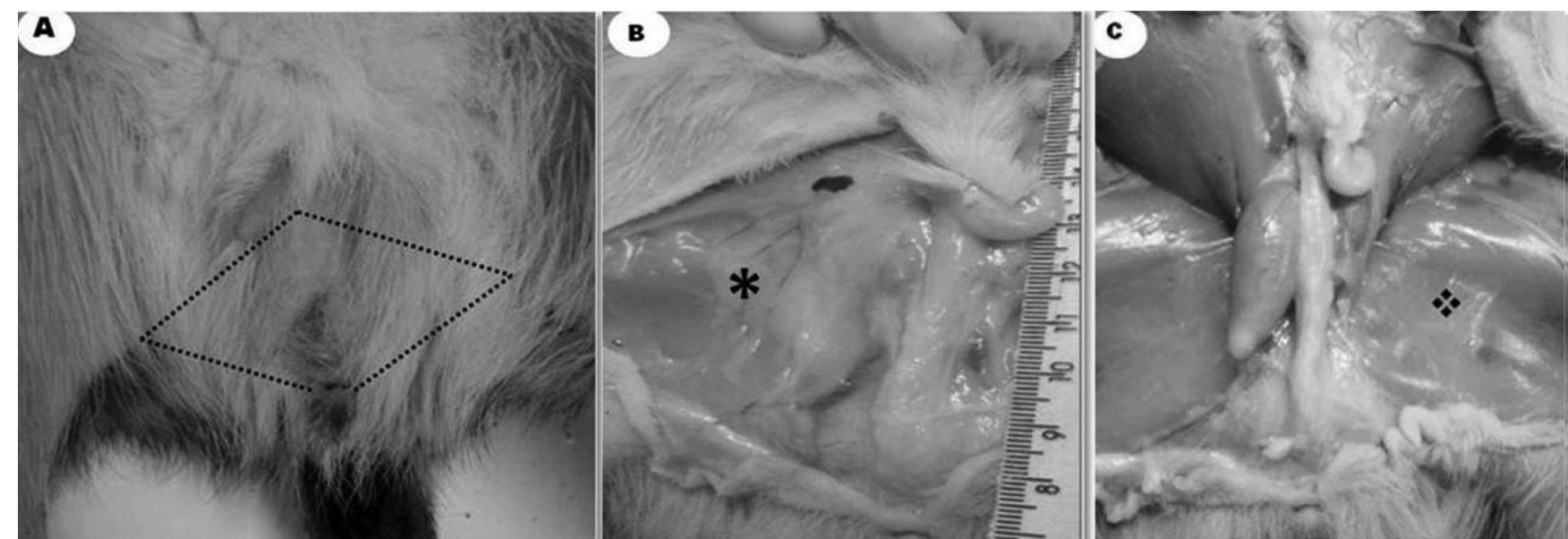

Fig.1. Vista ventral do períneo da viscacha. (A) Formato losangular do diafragma da pelve. (B) Fascia superficial do períneo. (C) Fáscia profunda.
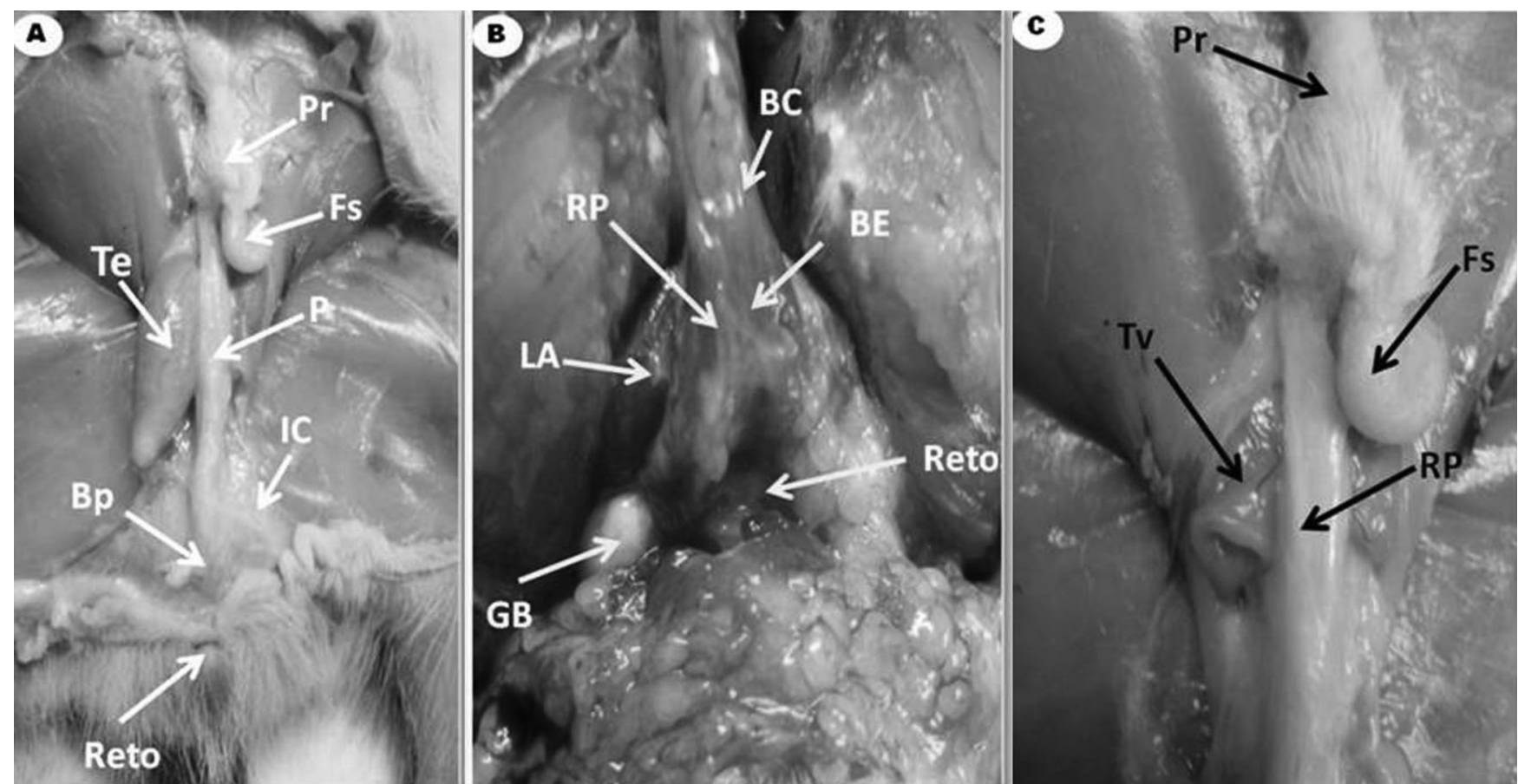

Fig.2, Vista ventral do períneo da viscacha. As figuras evidenciam a musculatura perineal formada pelos músculos: isquiocavernoso (IC), bulbocavernoso (BC). bulboesponjoso (BE); levantador do ânus (LA) e retrator do pênis (RP). (A) Testículo esquerdo (Te), pênis (P), prepúcio (Pp) e a localização do reto. (B) Glândula bubouretral. (C) Flexura sigmóide do pênis (Fs) e a túnica vaginal (Tv).

tricamente a partir do arco isquiático, formando a crura do pênis; M. bulbospongiosus, que se origina da parte cranial do bulbo e se insere na face caudal da raiz do pênis, e $M$. retractor penis, o qual segundo Ojasti (1973) em capivaras, que mediante uma contração, promove a ereção do pênis para o efeito de introdução na fêmea (Quadro 1).

Na Viscacha, M. ischiocavernosus origina-se na borda caudal da tuberosidade isquiática e da parte adjacente do ligamento sacrotuberal e está inserido sobre a "cruz" peniana, assim como no rato-topeira (Seney et al. 2009) e no coelho (Zempoalteca 2008). Este músculo em humanos tem como função fixar os ramos do pênis à pelve e auxiliar na ejaculação ao comprimir as veias dorsais do pênis, enquanto que em fêmeas de ratos ou camundongos (Hayes
1965, Cihfik et al. 1980, McKenna \& Nadelhaft 1986, Jordan 1997, Poortmans \& Wyndaele 1998, Tullber 1899), está ausente ou vestigial, porém está presente em ambos os sexos de rato-topeira africanos

M. retractor penis na viscacha é longo e liso, origina-se em ambos os lados do reto (Fig.2C), extende-se crânio-ventralmente ao longo da rafe mediana do períneo e se insere sobre a túnica albugínea na curva distal da flexura sigmóide (Fig.2C), como descreve Guisse (1994) em baleias; e diferentemente do relatado por Martins (2003) para preguiça-de-coleira, a qual destaca que o músculo retrator do pênis como bilateral com origem no ramo ventral do osso púbico. Entretanto na cutia, segundo Menezes et al. (2003), este músculo é denominado de subisquiocavernoso, pois me- 
Quadro 1. Músculos perineais da viscacha

\begin{tabular}{|c|c|c|}
\hline Músculos perineais & $\begin{array}{l}\text { Características } \\
\text { topográficas }\end{array}$ & $\begin{array}{l}\text { Ponto de } \\
\text { inserção }\end{array}$ \\
\hline Isquiocavernoso & $\begin{array}{l}\text { Origem na borda cau- } \\
\text { dal da tuberosidade } \\
\text { isquiática e da parte } \\
\text { adjacente do ligamen- } \\
\text { to sacrotuberal. }\end{array}$ & $\begin{array}{l}\text { Sobre a crua } \\
\text { peniana. }\end{array}$ \\
\hline Bulboesponjoso & $\begin{array}{l}\text { Origina da parte cra- } \\
\text { nial do bulbo do pênis. }\end{array}$ & $\begin{array}{l}\text { Face caudal da } \\
\text { raiz do pênis. }\end{array}$ \\
\hline Levantador do ânus & $\begin{array}{l}\text { Origem na sínfise pú- } \\
\text { bica acima do reto e } \\
\text { dorsal ao pênis. }\end{array}$ & \\
\hline Retrator do pênis & $\begin{array}{l}\text { Origem em cada lado } \\
\text { do reto e continua crâ- } \\
\text { nio ventralmente ao } \\
\text { longo da rafe mediana } \\
\text { do períneo. }\end{array}$ & $\begin{array}{l}\text { Sobre a túnica } \\
\text { albugínea na } \\
\text { curva distal da } \\
\text { flexura sigmói- } \\
\text { de. }\end{array}$ \\
\hline
\end{tabular}

diante sua contração, promove uma ereção do pênis para a introdução na fêmea no momento da cópula.

Entre M. ischiocavernosus ventral e o esfíncter anal em cães, encontramos $M$. bulbospongiosus, suas fibras são transversais, disposta sobre o bulbo do pênis e longitudinais distalmente sobre o corpo do pênis (Evans \& Delahunta 2001). Em viscachas M. bulbospongiosus é uma continuação do músculo uretral, assim como mostra Konig \& Liebich (2002) em seus estudos com animais domésticos; segundo este autor, animais com pênis fibroelástico, como no touro e o carneiro, este músculo apresenta-se restrito no terço proximal do pênis, como evidenciamos na viscacha.

M.bulbospongiosus na viscacha tem sua origem na parte cranial do bulbo e insere-se na fascia caudal da raiz do penis. Porém em alguns mamíferos como no gato (Martin et al.1974, Yamamoto et al. 1978, Ueyama et al. 1984); no rato (Schröder 1980, Katagiri et al. 1986, McKenna \& Nadelhaft 1986) e em Humanos (Yucel \& Baskin 2003), este músculo origina-se na túnica albugínea, lateral ao corpo do pênis e insere na fáscia do bulbo do pênis. Segundo Sachs (1982a;1983b) e Tang et al.(1998), este músculo apresenta um envolvimento no mecanismo de ereção, respostas reflexas a estimulaçaõ da região genital, participando do processo urogenital e expulsão das últimas gotas da urina ou sêmen da uretra (McKenna et al. 1991, Bancila et al. 1999) como nos ratos e na viscacha.

Na viscacha $M$. levator ani tem sua origem na sínfise púbica acima do reto; dorsal ao pênis apresenta-se como um músculo par, direito e esquerdo, que na margem anal demonstra-se com formato da letra " $V$ ", delimitando a fenda urogenital (Fig.2B); assim com em outros mamíferos e no homem, já descritos por Stromsten (1947) e Hall \& Brody (2001).

Sendo assim, destacamos que o estudo do assoalho pélvico em animais sejam domésticos ou silvestres é de grande valia, uma vez que este detalha a forma e conseqüentemente a função de cada músculo envolvido no mecanismo de ereção das distintas espécies. Estudos como este contribuem com o melhor entendimento dos mecanismos relacionados á ereção e ejaculação; ou seja, apresentam outra visão ao estudo da reprodução.

Agradecimentos.- À FAPESP (Fundação de Amparo a Pesquisa do Estado de São Paulo) pelo auxilio financeiro (Proc.10/10683-0), a CAPES (Coor- denação de Aperfeiçoamento de Pessoal de Nível Superior) pela bolsa de estudo.

\section{REFERÊNCIAS}

Aversi-Ferreira T.A., Vieira L.G., Pires R.M. \& Silva Z. \& Penhasilva N. 2006. Estudo anatômico dos músculos flexores superficiais do antebraço no macaco Cebus apella. Bioscience J. 22:139-144.

Bancila M., Verge D., Rampin O., Backstrom J.R., Sanders-Bush E., McKenna K., Marson L., Calas A. \& Giuliano F. 1999. 5-hydroxytryptamine2c receptors on spinal neurons controlling penile erection in the rat. Neuroscience 92:1523-1537.

Cihfik R., Gitmann E. \& Hanzllkovfi V.1970. Involution and hormone-induced persistence of the M. sphincter (Levator) ani in female rats. J. Anat. 106:93-100.

Dominguez S., Piezzi R.S., Scardapane L. \& Guzmán J.A.1987. A light e electron microscopic study of the pineal gland of the viscacha (Lagostmus maximus maximus). J. Pineal Res. 4:211-219.

Evans E. \& De Lahunta A. 1994. Guia para a Dissecção do Cão. 3ª ed. Guanabara Koogan, Rio de Janeiro. 206p.

Flamini M.A., Barbeito C.G., Gimeno E.J. \& Portiansky E.L. 2009. Histology, histochemistry and morphometry of the ovary of the adult plains viscacha (Lagostomus maximus) indifferent reproductive stages. Acta Zool. 90:390-400

Flamini M.A., Portiansky E.L., Favaron P., Martins D., Ambrósio C., Mess A., Miglino M.A., Barbeito C.G. 2011. Chorioallantoic and yolk sac placentation in the plains viscacha (Lagostomus maximus): A caviomorph rodent with natural polyovulation. Placenta 32(12):963-968. doi:10.1016/j. placenta.2011.09.02.

Franciolli A.L.R., Ambrósio C.E., Oliveira M.F., Morini A.C., Favaron P.O., Machado M.R.F. \& Miglino M.A. 2011. Os histricomorfos sul-americanos: uma análise comparativa do desenvolvimento embriológico. Pesq. Vet. Bras. 31:441-446.

Guisse S.D., Bisaillon A., Seguin B. \& Lagacé A. 1994. The anatomy of the male genital system of the beluga wale, Delphinapterus leucas, with special reference to the penis. Anat. Histol. Embryol. 23:207-216.

Gray D.,Gardner E. \& O'rahilly R. 1988. Anatomia: estudo regional do corpo

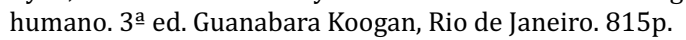

Hart B.1972. The action of extrinsic penile muscles during copulation in the male dog. Anat. Rec. 173:1-6.

Hart B.L. \& Melese-D’hospital P.Y. 1983. Penile mechanisms and the role of the striated penile muscles in penile reflexes. Physiol. Behav. 31:807.

Hayes K. 1965. Yhe so-called 'Levator ani' of the rat. Acta Endocrinol. 48:337-347.

Hart B.L. \& Melese-D'hospital P.Y. 1983. .Penile mechanisms and the role of the striated penile muscles in penile reflexes. Physiol. Behav. 31:807813.

Hall Carrie E. \& Brody L.T. 2001. Exercício Terapêutico: na busca da função. Guanabara Koogan, Rio de Janeiro. 107p.

Holmes G.M. \& Sachs B.D. 1994. Physiology and mechanics of rat Levator ani muscle: Evidence for a sexual function. Physiol. Behav. 55:255-266.

Jordan C. 1997. Androgen receptor (Ar) immunoreactivity in rat pudendal motoneurons: implications for accessory proteins. Horm. Behav 32:110 .

Jackson J. 1989. Reproductive parameters of the planis viscacha (Lagostomus maximus) in San Luis Province, Argentina. Vida Silvestre Neotropical 2:57-62.

Karacan I., Aslan C. \& Hirshkowitz M.1983. Erectile mechanism in man. Science 220:1080-1082.

Konig H.E. \& Liebich H.G. 2002. Anatomia dos Animais Domésticos. Artmed, São Paulo. 308p.

Katagiri T., Gibson S.J., Su H.C. \& Polak J.M. 1986. Composition and central projections of the pudendal nerve in the rat investigated by combined peptide immunocytochemistry and retrograde fluorescent labelling. Brain Res. 372:313-322. 
Yajima M. 1989. effects of vasoactive drugs on isolated rabbit Corpus cavernosum penis. Jpn. J. Urol. 80:1422-142.

Llanos A.C. \& Crespo J.A. 1952. Ecología de la vizcacha (Lagostomus maximus maximus) in Nordeste de la Provincia de Entre Rios. Revta Invest. Agricolas 6:289-378.

McKenna K.E. \& Nadelhaft I. 1986. The organization of the pudendal nerve in the male and female rat. J. Comp. Neurol. 248:532-549.

Mohamed F., Fogal T., Dominguez S., Scardapane L., Guzman J. \& Piezzi R. 1994. Seasonal variations and utrastructure of coloid accumulation in the pituitary Pars distalis of vizcacha (Lagostomus maximus maximus). Biocell 18:23-31.

Manzanares M.A. 2007. Adaptaciones musculares relacionadas a áreas corporales que participan activamente en el vuelo de Anhima cornuta (Aves: Anseriformes, Anhimidae). Revta Peru. Biol. 14:145-150.

Martins D.S. 2003. Morfologia do sistema reprodutor masculino da preguiça-de-coleira (Bradypus Torquatus Illiger, 1811). Dissertação Programa de Pós-Graduação em Animais Domésticos e Silvestres, Faculdade de Medicina Veterinária e Zootecnia, Universidade de São Paulo, São Paulo. $116 \mathrm{p}$.

Moore K.L. \& Dalley A.F. 1994. Anatomia: orientando para a clínica. 3ª̣ ed. Guanabara Koogan Rio de Janeiro. 1142p.

Mckenna K.E., Chung S.K. \& Mcvary V. 1991. A model for the study of sexual function in anesthetized male and female rats. Am. J. Physiol. 261:12761285.

Menezes A.J.D., Carvalho M.A.M., Assis Neto C.A., Oliveira F.M., Farias C.E., Miglino M.A. \& Medeiros X.G. 2003. Morfologia dos órgãos genitais externos do macho de cutia (Dasyprocta aguti Linneus, 1766). Braz. J. Vet. Res. Anim. Sci. 40:148-153.

Onuf B. \& Collins J. 1900. On the arrangement and function of the cell groups of the sacral region of the spinal cord in the man. Arch. Neurol. Psychopathol. 3:387-412.

Ojasti J. 1973. Estudio Biológico del Chigüira o Capibara. Ediciones del Fondo Nacional de Investigaciones Agropecuárias, Caracas. 275p

Piezzi R.S., Cavicchia J. \& Scardapane L.1985. Marginal cells of the viscacha pituitary left: a correlate study by thin-sectioning, lanthanum inter cellular teacer and freeze-fracture techniques. Biol. Cell. 322:193203.

Piezzi R.S., Guzmán J.A., Pelzer L.E., Scardapane L. \& Dominguez S. 1984. Biological role of the neal responses to the environmental photoperiod. Arch. Biol. Med. Exp., Santiago, 17:273-282.
Poortmans A. \& Wyndaele J.J. M.1998. Levator ani in the rat: Does it really lift the anus. Anat. Rec. 251:20-27.

Seney M.L., Kelly D.A., Bruce D. Goldman B.D., Umbera R.S. \& Forger N.G. 2009. Social structure predicts genital morphology in African mole-rats. PLoS ONE 4:7477

Sachs B.D. 1982. Role of striated penile muscles in penile reflexes, copulation and induction of pregnancy in the rat. J. Reprod. Fertil. 66:433-443.

Sachs B.D. 1983. Potency and fertility: hormonal and mechanical causes and effects of penile actions in rats, p.86-110. In: Balthazart J., Prve E. \& Gilles R. (Eds), Hormones and Behaviour in Higher Vertebrates. Springer-Verlag, Berlin.

Sachs B.D.1982. Role of striated penile muscles in penile reflexes, copulation and induction of pregnancy. J. Reprod. Fertil. 66:433-443.

Stromsten F. 1947. Mammalian Anatomy.17 ${ }^{\text {th }}$ ed. Blakiston, Philadelphia, p.142-144.

Schroder H.D. 1980. Organization of the motoneurons innervating the pelvic muscles of the male rat. J. Comp. Neurol. 192:567-587.

Tullberg T. 1899. Über das System der Nagethiere: Eine phylogenetische Studie. Nov. Acta. Reg. Soc. Sci. Up 3:1-514.

Tang Y., Rampin O., Calas A., Facchinetti P. \& Giuliano F. 1998. Oxytocinergic and serotonergic innervation of identified lumbosacral nuclei controlling penile erection in the male rat. Neuroscience 82:241-254.

Ueyama T., Arakawa H. \& Mizuno M. 1987. Central distribution of efferent and afferent components of the pudendal nerve in rat. Anat. Embriol. 117:37-49.

Zempoalteca R.A. \& Lucio J.R. 2008. Perineal striated muscles: anatomy, spinal motoneurons, and participation on copulatory behavior in male rabbits (Oryctolagus cuniculus). Synapse 62:653-661.

Yamamoto T., Satomi H., Ise H., Takatama H. \& Takahashi K. 1978. Sacral spinal innervations of the rectal and vesical smooth muscles and the sphincteric striated muscles as demonstrated by the horseradish peroxidase method. Neurosci. Lett. 7:41-47.

Yucel S. \& Baskin L.S. 2003. Neuroanatomy of the male urethra and perineum. BJU Int. 92: 624-630

Watanabe Li-Sei 2000. Erhart: elementos de anatomia humana. 9a ed. Atheneu, São Paulo. 243p.

White N.R., Cagiano R. \& Barfield R.J. 1990. Receptivity of the female rat (Rattus norvegicus) after male devocalization: a ventral perspective. J. Comp. Psychol. 104:174-151. 